\title{
Implicancias éticas y políticas de la intervención social pos-dictadura en Chile
}

\author{
Walter Manuel Molina Chávez*
}

\begin{abstract}
RESUMEN
La sociedad chilena de las últimas dos décadas enfrentó múltiples desafíos, uno de los más urgentes y transversales fue lograr mayores niveles de justicia, equidad e integración social en el Chile pos-dictadura. Junto con estas demandas surgen una serie de otros desafíos en el ámbito de problemas sociales emergentes que dan origen a intervenciones sociales complejas. Por una parte, promover mayores niveles de integración e inclusión social, y por otra, favorecer la capacidad de acción de los actores sociales desde sus espacios cotidianos en los cuales es posible promover nuevas formas de sociabilidad y gestión social de sus demandas. En otras palabras, la intervención social contemporánea se enfrenta al desafío permanente de contribuir a la generación de nuevos horizontes de sentido ético y político.
\end{abstract}

Palabras clave: Intervención social - Contexto de pos dictadura Implicancias éticas y políticas - trabajo social.

\section{Implicâncias éticas e políticas da intervenção social pós-ditadura no Chile}

\author{
RESUMO
}

A sociedade chilena das últimas duas décadas enfrentou diversos desafios, um dos mais urgentes e transversais foi lograr maiores níveis de justiça, equidade e integração social no Chile pós-ditadura. Junto com estas demandas, surgiu uma série de outros desafios no âmbito de problemas sociais emergentes que dão origem a intervenções sociais complexas. Por uma parte, promover maiores níveis de integração e inclusão social, e por outra, favorecer a capacidade de ação dos atores sociais desde seus espaços cotidianos nos quais é possível promover novas formas de sociabilidade e gestão social de suas demandas. Em outras palavras, a intervenção social contemporânea se enfrenta ao desafio permanente de contribuir a geração de novos horizontes de sentido ético e político.

Palavras chave: Intervenção social - Contexto de pós-ditadura Implicâncias éticas e políticas - trabalho social.

* Chileno, Trabajador Social, Dr. en Ciencias de la educación, académico Escuela de Trabajo Social. Universidad de Magallanes.Correo electrónico: walter.molina@umag.cl 


\title{
Ethical and political implications of social intervention in the post-dictatorship Chile
}

\begin{abstract}
Chilean society of the past two decades faced many challenges; one of the most urgent and cross-sectional was to achieve higher levels of justice, equity and social integration in post-dictatorship Chile. Along with these demands, there have been a number of other challenges which have risen in terms of emerging social issues requiring complex social interventions. Thus, strategies to deal with these problems should aim, on the one hand, to promote higher levels of integration and social inclusion, and on the other, to empower social actors from their everyday spaces in which it is possible to generate new forms of sociability and social management of their demands. In other words, the contemporary social intervention faces the ongoing challenge of contributing to the development of new horizons of ethical and political sense.
\end{abstract}

Keywords: Social Intervention - Post-Dictatorship Context - Ethical and Political Implications - Social Work

\section{Introducción}

Este artículo es una reflexión sobre la intervención social en un período específico de la historia social y política de nuestro país que puede definirse como "pos-dictadura en Chile". Esta forma de nombrar el contexto político, económico, social y cultural, no es inocente, por cuanto alude a ciertos elementos que enmarcan y condicionan fuertemente la puesta en escena del Trabajo Social en el Chile contemporáneo.

Cabe señalar que este período de transición de una dictadura militar (1973-1989) a un sistema democrático (1990-2010), a su vez, se realiza en los intersticios del tránsito del Siglo XX al Siglo XXI. En consecuencia, se trata de un periodo marcado por una doble transición. Por una parte, se producen significativos cambios en la institucionalidad política del país y se recuperan parcialmente algunas formas e instituciones democráticas que sustentan la vida social. Y por otra, se producen profundos cambios en todas las esferas de la sociedad: economía, política, re-composición de las clases sociales y la emergencia de nuevos imaginarios socioculturales. Todo ello tiene una serie de consecuencias concretas para los procesos de intervención social en general y específicamente para el Trabajo Social. 
Más allá de las diversas aproximaciones y balances históricos que se puedan hacer en torno a lo ocurrido en las dos últimas décadas en Chile, el aporte principal de ese periodo, en el plano político, consiste en que se restituyó un sistema político democrático que había sido negado a partir del golpe militar del año 1973. Por lo tanto, se re-configura una institucionalidad política y social que, esencialmente, repone las normas y valores de la convivencia democrática en el país, aún cuando se trata de una "recuperación democrática parcial", por cuanto se conservan elementos y mecanismos claramente autoritarios en la organización de la vida social (como consecuencia de la conservación de la Constitución Política de 1980). No obstante, esta recuperación de la democracia se constituye en un hito importante para la re-configuración tanto de "lo político" como de "lo social" a partir de la década de los noventa. Este hecho político da una impronta a las intervenciones sociales que se realizan en el período. Es decir, se trata de una intervención que se enmarca en el progresivo re-conocimiento de los derechos de ciudadanía a los sujetos y actores sociales que conforman la sociedad chilena. Esta ciudadanía es entendida como un progresivo despliegue/ apropiación de derechos políticos, sociales y culturales que habían sido denegados y vulnerados en el periodo dictatorial.

Otro aspecto que marca el escenario de intervención social contemporánea en Chile, es el cambio de épocas (y de siglos) que diversos autores (Castells, 2003; Bajoit, 2003; 2009) han caracterizado como el tránsito de una sociedad industrial a una sociedad de la información o post industrial. A juicio de Bajoit (1999) en el marco de este tránsito de un tipo de sociedad a otro, se produce una verdadera "mutación cultural", es decir, se verifica el paso a un Modelo Cultural de la Razón Social, el cual es propio de las sociedades industriales avanzadas y que se constituye en torno a un conjunto de principios de sentido que lo legitiman: prominencia de "lo colectivo" por sobre lo individual, valorización de la ciencia como modo de racionalización de la vida, y el valor del progreso o modernización como factores clave del bienestar social y del mejoramiento de la calidad de Vida. Este modelo imperó en el mundo capitalista occidental hasta aproximadamente principios de la década del '70. Por otra parte, para el mismo autor (Bajoit, 1999), en el Chile de la transición a la democracia, comienza a emerger un Modelo Cultural Identitario (el cual se ha manifestado en Europa desde comienzos de los años setenta) y cuyo centro de referencia es el propio individuo y 
su urgente deseo de realización personal y autonomía, es decir, una tendencia a mejorar calidad de vida a partir de sí mismos, es decir, una tendencia al logro de una auto realización autónoma. Lo anterior genera una segunda impronta y nuevos desafíos para la intervención social que se realiza en Chile en los últimos veinte años: ¿Cómo intervenir en un contexto sociocultural en el cual el sujeto despliega su necesidad de libertad individual, autonomía y sus deseos de realización personal?

Finalmente, es necesario destacar un elemento transversal del escenario social pos-dictadura y que se despliega como telón de fondo de los procesos de intervención social en el país. Este alude a uno de los rasgos más expresivos de la sociedad chilena actual: la instalación de la desigualdad social en el corazón de las relaciones sociales en el Chile contemporáneo. Lo anterior, se relaciona con la puesta en escena de un modelo de desarrollo económico/social de tipo capitalista, neoliberal y globalizado. Y cuya implementación contó con un relativo consenso en todas las fuerzas políticas que estuvieron en juego en el periodo de la transición a la democracia en Chile. Lo anterior tuvo como resultado una profundización de la concentración de la riqueza y el aumento de la desigualdad social, configurando un escenario económico-social-cultural paradójico; que por una parte seduce a una gran parte de la población chilena y por otro lado los rechaza o excluye de los bienes públicos más relevantes para la vida: salud, educación, seguridad social y trabajo de calidad.

Es así como en el curso de los últimos años, los datos muestran un desarrollo "objetivo" en lo económico y social (altas tasas de crecimiento económico, aumento del ingreso per cápita de los chilenos, mejoramiento de indicadores en salud y educación, etcétera), un escenario de estabilidad política y modernizaciones de diferentes esferas de la vida social. Pero por otra parte, a nivel de la percepción subjetiva de la ciudadanía se observa un creciente malestar sociocultural frente a una tendencia sostenida a la exclusión y fragmentación de la sociedad chilena. A pesar del fuerte desarrollo económico experimentado durante la década del noventa, en el Informe de Desarrollo Humano (2002) se sostiene que "más de la mitad de los encuestados por dicho estudio, se ven a sí mismos como un perdedor y en términos globales un 75\% de los encuestados manifiesta sentimientos negativos acerca del sistema económico" (PNUD, 2002:41).

En este contexto de creciente malestar sociocultural de los diversos actores sociales con la escandalosa desigualdad social 
en Chile, el rector de la Universidad Alberto Hurtado ${ }^{1}$ ha señalado recientemente que la sociedad chilena actual es una sociedad profundamente fragmentada, agregando que en nuestro país hay dos o más Chile y con ello, coexisten varios mundos sociales que no entran en contacto entre sí y en consecuencia, es el alma de Chile la que se ha fracturado. Entonces, surge la imprescindible pregunta: ¿cómo la intervención social se hace cargo de esa diversidad de mundos, de esas realidades separadas por un abismo de injusticias e inequidades sociales?

\section{Contexto económico-social y sus implicancias ético-políticas para la definición de estrategias de intervención social}

El Chile de pos-dictadura ha devenido en una sociedad profundamente fragmentada en lo económico, social, político y cultural. En el seno de las relaciones sociales se han instalado (incluso logrando hegemonía cultural) diversos y eficaces mecanismos de exclusión social (segmentación de los mercados de la educación, salud, vivienda, entre otros). La principal evidencia de la consolidación de la desigualdad social en Chile hace referencia a los procesos de concentración de la riqueza y concretamente la desigual distribución de los ingresos autónomos entre los hogares de nuestro país.

En este contexto, es relevante observar los resultados de la encuesta CASEN (MIDEPLAN, 2007), respecto de la evolución en la distribución del ingreso autónomo (aquellos que generan los hogares por sus propios medios) y que evidencian una leve mejoría en el periodo histórico que estamos analizando:

\begin{tabular}{|l|r|r|r|r|r|r|r|r|}
\hline Decil & 1990 & $\mathbf{1 9 9 2}$ & $\mathbf{1 9 9 4}$ & $\mathbf{1 9 9 6}$ & $\mathbf{1 9 9 8}$ & $\mathbf{2 0 0 0}$ & $\mathbf{2 0 0 3}$ & $\mathbf{2 0 0 6}$ \\
\hline I & 1,4 & 1,5 & 1,4 & 1,3 & 1,2 & 1,3 & 1,2 & 1,2 \\
\hline II & 2,7 & 2,8 & 2,7 & 2,6 & 2,5 & 2,7 & 2,7 & 2,9 \\
\hline III & 3,6 & 3,7 & 3,5 & 3,5 & 3,5 & 3,6 & 3,6 & 3,9 \\
\hline IV & 4,5 & 4,7 & 4,5 & 4,5 & 4,5 & 4,5 & 4,7 & 4,9 \\
\hline V & 5,4 & 5,6 & 5,6 & 5,4 & 5,3 & 5,7 & 5,4 & 5,6 \\
\hline VI & 6,9 & 6,6 & 6,4 & 6,3 & 6,4 & 6,2 & 6,6 & 7,0 \\
\hline VII & 7,7 & 8,1 & 8,1 & 8,2 & 8,3 & 7,9 & 8,2 & 8,7 \\
\hline VIII & 10,4 & 10,5 & 10,6 & 11,1 & 11,0 & 10,4 & 10,7 & 11,1 \\
\hline IX & 15,2 & 14,8 & 15,4 & 15,4 & 16,0 & 15,1 & 15,3 & 16,0 \\
\hline X & 42,2 & 41,8 & $\mathbf{4 1 , 8}$ & $\mathbf{4 1 , 8}$ & 41,4 & 42,7 & 41,5 & 38,6 \\
\hline Total & 100,0 & 100,0 & 100,0 & 100,0 & 100,0 & 100,0 & 100,0 & 100,0 \\
\hline
\end{tabular}

Fuente: Mideplan en base a Encuesta CASEN, años respectivos.

S.J. Padre Fernando Montes, en la apertura del Seminario: "Palabras y cosas en Trabajo Social. El lugar de las estrategias de intervención". 28 de mayo, 2009. 
Como se puede observar, específicamente durante el periodo del año 2003 al 2006 se produce un leve aumento en la participación del ingreso autónomo para los deciles II al IX. En cambio, el primer decil (10\% de menores ingresos) mantiene inalterada su participación que es de 1,2\%. En una posición distinta se encuentra el decil X (10\% de los hogares de más altos ingresos) que por primera vez cae su participación en el ingreso autónomo desde $41,5 \%$ en 2003 a un $38,6 \%$ en 2006. Tal vez esta fue una pequeña luz de esperanza, que muestra que es posible mejorar las condiciones de vida de la población y particularmente de los hogares de menores ingresos.

Sin embargo, en términos globales, los datos de la encuesta CASEN (2006) confirman que la distribución del ingreso autónomo continua siendo altamente desigual en el país. En términos del índice y coeficiente de Gini se muestra que el 20\% de los hogares con mayores ingresos aún concentran el 54,6\% del total de los ingresos autónomos, en tanto que el 20\% de hogares de menores ingresos reciben sólo el $4.1 \%$ de estos ingresos. De este modo, la diferencia de ingresos entre un hogar pobre y un hogar rico en el Chile contemporáneo, es de 13.1 veces más a favor de los sectores de mayores ingresos monetarios. La variación puede apreciarse en el siguiente gráfico:

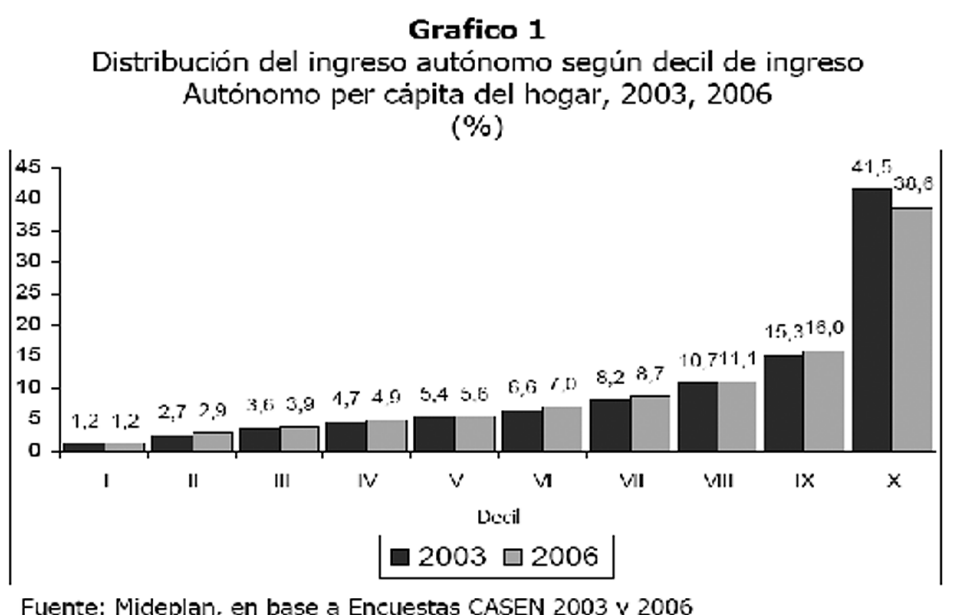

Fuente: Mideplan, en base a Encuestas CASEN 2003 y 2006

Cabe señalar que toda esta secuela de desigualdad e inequidades sociales han sido mitigadas sólo parcialmente y focalizadamente por el Sistema de Protección Social introducido en el gobierno de Michelle Bachelet (2006-2010). 
En un contexto más amplio de análisis, y específicamente en el plano económico, se observa que los mecanismos de mercado se consolidan como la forma privilegiada de asignar los recursos sociales/públicos, y aquello se ha transformado en el plano social, en el modo más eficiente de generar formas de exclusión social. Ello, por cuanto a través de esta vía (del mercado) se introducen potentes mecanismos de segmentación de la oferta de bienes y servicios que son claves para generar mejores condiciones de vida de la población chilena. Por ejemplo, en el caso de la oferta educativa se llega a consolidar una realidad dramática (e inaceptable) de la existencia de escuelas para "ricos" y escuelas para "pobres" (colegios particulares pagados, subvencionados y municipales). Otro ejemplo evidente, lo constituye la diferenciación de la oferta que proporcionan los servicios de salud públicos y privados. Del mismo modo, la configuración de un mercado inmobiliario bajo el control de actores privados, terminó por consolidar los procesos de re-localización socio-espacial de la pobreza urbana en los principales centros metropolitanos de nuestro país. Como se puede observar, las pruebas y evidencias empíricas de la segmentación social y económica de la sociedad chilena son múltiples y profundas.

En este contexto de desigualdad social, surge uno de los principales desafíos éticos y políticos para la intervención social, es decir, la necesidad de contribuir a generar mayores grados de igualdad/equidad social que posibilite el desarrollo de todos sus miembros/ciudadanos. De este modo, el lugar de las estrategias de intervención social contemporánea, particularmente desde Trabajo Social, se define a partir de una doble demanda. Por una parte, promover mayores niveles de integración/inclusión social (dimensión sistémica) y desde otro ámbito, potenciar la integración social desde la base social, desde la acción social de los actores en sus espacios cotidianos y particularmente desde los territorios locales en los cuales es posible promover los espacios de sociabilidad como son las redes de apoyo mutuo y de reciprocidad social. Es decir, se trata de potenciar las relaciones comunitarias como mecanismo de restauración de la confianza social en Chile. Evidentemente, ello debe ir acompañado con mejorar aspectos claves de la calidad de vida de los chilenos en los siguientes aspectos: a) mejorar sustantivamente la retribución al trabajo asalariado, b) elevar las condiciones laborales, c) proveer servicios educativos y de salud de calidad; entre otros aspectos relevantes para el desarrollo humano. 
La articulación de los procesos de integración social sistémica (desde arriba) y la integración social desde la base social puede constituirse en el horizonte de sentido ético y político de la intervención social contemporánea.

\section{Desafíos para las políticas públicas y la intervención social en Chile pos-dictadura}

En el escenario de las inequidades sociales antes descritas, durante los últimos veinte años de intervención social en Chile, emergió un importante desafío para la teoría/práctica del Trabajo Social contemporáneo: producir conocimiento/acción en torno a los diversos mecanismos de (re)producción de las desigualdades sociales en Chile. Junto con ello, se trató de generar propuestas y estrategias de intervención social que potencien la humanización de diversos espacios de la vida social y de las posibilidades de realización de la ciudadanía moderna: la participación ciudadana, los espacios educativos, familia, el barrio, el trabajo, escuela, entre otros espacios potenciales.

En este contexto, el esfuerzo en pos del mejoramiento de la equidad y calidad de los servicios educativos ocupó un lugar relevante en las últimas dos décadas en Chile. Sin embargo, el balance actual arroja un sistema educativo altamente segmentado y con niveles de calidad diferenciados por estrato socioeconómico y con bajos resultados de aprendizaje. Al respecto son expresivos los resultados que obtuvieron los estudiantes chilenos que rindieron

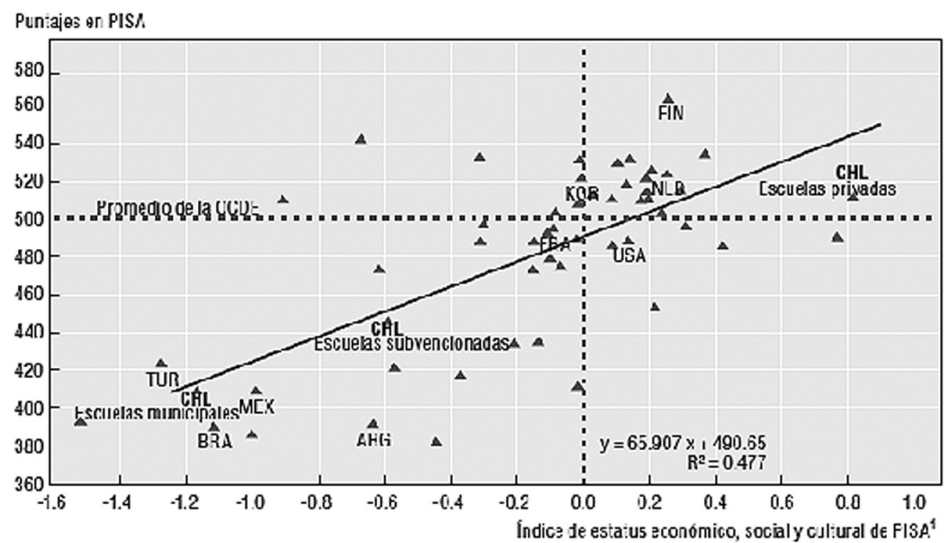

1. El indice de estatus econ $\delta$ mico, social y cultural de PISA (ESCS) sintetiza varios aspectos del trasfoncio socio-económico, entre los que se encuentran el nivel de escelaridad de los padres, su categoría profeeional y el acceso de los alumnos a los recureos educativos. Se normaliza a 0 para la media de la CC.DF.. Un valor de indice maynr es sinónimn de un contexto socio-eccnómico más plpradn. Fusnte: OCDE Rcsultados de PISA 2006. 
la prueba PISA (2006) y las diferencias de puntajes (mínimo 360 y máximo 580 puntos) entre escuelas municipales, particulares subvencionadas y particulares pagadas en Chile.

Como muestra la imagen anterior, los resultados obtenidos en el Programa Internacional para la Evaluación de Estudiantes de la OCDE (PISA, 2006) ${ }^{2}$. En todas estas mediciones Chile ha logrado bajos resultados en las tres áreas que mide este instrumento: Matemáticas, ciencias y lectura. Los resultados nuevamente indican que pese a que Chile ha mostrado un incremento significativo en lectura entre los años 2000 y 2006 (+33 puntos), aún se ubica bajo el puntaje promedio que logran el conjunto de países del OCDE (2006). En este contexto general de análisis, nuestro país alcanza 442 puntos frente al promedio obtenido por los países del OCDE el cual es de 492 puntos; esta distancia se incrementa significativamente si nos comparamos respecto de los países que alcanzan los puntajes más altos. Y la situación más grave que evidencian estos resultados es la diferencia abismal de puntajes que alcanzan en dicha prueba los estudiantes de escuelas municipales, particulares subvencionadas y particulares pagadas.

Uno de los aspectos agravantes de esta realidad educativa segmentada por ingresos socioeconómicos presentada en el gráfico anterior, es que tal como señala Casassus (2003), existe una fuerte interacción entre las desigualdades en los ingresos monetarios de los hogares y la configuración de las otras desigualdades sociales, entre las cuales se encuentran las desigualdades educativas. Como señala el mismo autor, las desigualdades en educación, a su vez, repercuten en la distribución de los ingresos, generando con ello un círculo vicioso de desigualdades en la sociedad. En este escenario de desarrollo económico y social contradictorio en el caso de Chile; la educación (y el sistema educativo en su conjunto) emerge como una potente herramienta que puede contribuir significativamente a superar esta situación de desigualdad en la sociedad chilena, aun cuando no puede corregir por sí sola toda la desigualdad de base de una sociedad. No obstante, las políticas públicas educativas pueden contribuir a remover los factores que (re)producen dicha desigualdad entre los distintos sectores de la población chilena. En esa perspectiva, lo educativo emerge como un espacio

2 Programa para la Evaluación Internacional de Alumnos. PISA por su nombre en inglés: Programme for International Student Assessment. 
propicio para implementar nuevas estrategias de intervención social con un alto impacto social. Y de este modo, revitalizar el natural vínculo que existe entre Educación (no escolarización) y Trabajo Social. Tal vez ello nos da la oportunidad de gestar intervenciones socioeducativas que aporten significativamente a mejorar/superar las desigualdades en el campo de la educación en el Chile de las próximas décadas. Desde el ámbito educativo, se configura un desafío ético-político específico en torno a la constitución de un saber profesional/disciplinario que ubique a Trabajo Social al servicio de la construcción de un país más justo, más equitativo y más integrado desde el punto de vista económico, social, político y cultural.

\section{Algunos elementos para (re)pensar los desafíos de la intervención social}

En el contexto del análisis presentado y considerando la deriva en la que se encuentra el Chile actual, en nuestro país surge una demanda radical por integración e inclusión social, tanto a nivel de una mayor participación de todos los actores sociales en los beneficios del desarrollo económico, así como en relación a la necesidad de generar condiciones para democratizar las relaciones sociales; posibilitando que éstas sean cada vez más transversales, es decir, más diversas, heterogéneas y que permitan superar el profundo miedo al otro, a la alteridad. Y en este contexto, esta demanda por mayor integración social es el telón de fondo de todos los procesos de intervención social que se realizan en nuestra sociedad en la actualidad. En este sentido, para algunos actores institucionales (CEPAL 2009; PNUD, 2002), se trata de una demanda por mayor cohesión social, es decir, por la necesidad de re-construir el vínculo social que ha sido fracturado por los procesos de creciente desigualdad social y los mecanismos de exclusión social antes descritos. Para otros, en cambio, se trata de la necesidad de re-establecer la integración social (sistémica), la inclusión social (por la vía de mejorar el acceso a bienes y servicios a través de la política social), la confianza social, el diálogo y el contacto en las relaciones sociales entre los distintos actores de la sociedad chilena. En cualquiera de los enfoques antes señalados, esa demanda por mayor integración social (a condición de superar los actuales niveles de desigualdad social) es el escenario obligado en el cual se desarrollan los procesos de intervención social tanto a nivel individual, grupal, comunitario, organizacional y/o institucional, 
emergiendo allí un potente desafío para la teoría/práctica del Trabajo social.

En dichas condiciones contextuales, es donde se configura un horizonte de sentido (en tanto lugar simbólico) para las estrategias de intervención social contemporánea en Chile. Es decir, ese lugar o intersticio donde se anida una de las finalidades de la intervención social y que tiene relación con la necesidad de contribuir significativamente a la articulación de la dimensión sistémica de los procesos de desarrollo y de la dimensión subjetiva de estos. La necesidad de promover procesos de integración social por arriba (dimensión sistémica del desarrollo) y donde las políticas públicas pueden contribuir significativamente a frenar los procesos de desigualdad social y fragmentación de la sociedad chilena. Pero por otra parte, la intervención social debe contribuir a promover una integración social por abajo, es decir, desde la acción social de los actores y la promoción de los vínculos comunitarios entre ellos, la configuración de redes de apoyo y sobre todo el empoderamiento político y social de las comunidades y su movilización en pos del desarrollo humano en sus propios territorios. Precisamente uno de los dramas del desarrollo económico/humano "logrado" en la sociedad chilena en los últimos 20 años, es su no integración de la dimensión subjetiva y la no superación de los riesgos, incertidumbres y amenazas "percibidas" por los actores y que se activan con la profundización de un modelo de desarrollo capitalista en su versión neoliberal globalizada en nuestro país.

Por otra parte, frente a la pregunta por los desafíos éticos/ políticos de la intervención social actual, es relevante enfatizar que el principal desafío implica mostrar significativamente desde nuestros espacios disciplinarios/profesionales, cuáles son los principales mecanismos de configuración de la exclusión social. Ello implica estudiar cómo operan dichos mecanismos, indagar en los distintos campos de conformación de las problemáticas sociales contemporáneas y proponer estrategias de intervención complejas para enfrentar fenómenos sociales emergentes. De acuerdo a esto cabe realizar la pregunta: ¿por qué enfatizar en lo ético-político? Porque, por una parte estos desafíos aluden a la necesidad de que los actores sociales incorporen la dimensión política en sus acciones orientadas al mejoramiento de condiciones de vida. Y por otro lado, ello constituye un desafío político en sí mismo, en tanto se relaciona con el ejercicio del poder. Y en este sentido, las estrategias de intervención social son 
un tipo de relación social específica que implica un determinado posicionamiento/poder de los actores sociales concernidos en dicha relación. En la otra cara de la intervención, somos actores sociales/ciudadanos y en consecuencia, tenemos la capacidad de influir en otros sujetos; por lo tanto, de ahí surge una demanda y un desafío para la intervención social que básicamente se relaciona con contribuir a la re-configuración de "lo social" sin exclusiones, sin fragmentación y promoviendo la autonomía de los sujetos. En términos generales, se trata de lograr una hegemonía cultural que gane la batalla de la exclusión social en el plano de la cultura, de las ideas y más precisamente de las prácticas culturales. Es decir, construir una hegemonía cultural que se manifieste como rechazo ético/político a cualquier mecanismo social que promueva zonas de exclusión social y segregación social en nuestras sociedades.

Una de las conclusiones preliminares, en esta línea de los desafíos éticos-políticos que nos interpela a (nos)otros cotidianamente como profesionales y como personas/ciudadanos, es que si la ética tiene una marca originaria, ese sello indeleble es el desafío de ser sujetos y actores sociales simultáneamente. Lo cual en términos sociológicos significa, tener la capacidad de constituirnos en sujetos (individuales) con capacidad de influir tanto en "sí mismo" y, por otra parte, influir en "otros sujetos" (colectivos). Y desde esta doble condición de sujeto/actor discernir y contribuir a ampliar los ámbitos de la justicia, de lo que es correcto, y, en definitiva, tomar una posición ética y política que signifique un avance para el desarrollo humano en nuestro país.

Finalmente, frente a los actuales contextos de exclusión social, emerge una nueva interpelación ética-política para la intervención social; la cual nos obliga a tomar posicionamiento como profesionales de la intervención, frente a matrices teóricas, metodológicas, epistemológicas que permitan una comprensión compleja de la realidad social y que respondan a los múltiples desafíos de la intervención social que impulsamos desde nuestros espacios profesionales y disciplinarios.

Desde nuestra perspectiva, son los modos de habitar el territorio, el espacio, la ciudad, el que nos interpela cotidianamente para implementar una intervención social. Ese lugar social que nos interpela, puede ser un entorno deteriorado, un espacio público conquistado por la "delincuencia", una plaza destruida por la acción de los propios vecinos, en fin, un espacio concreto donde 
se ubica el actor y desde el cual dialoga cotidianamente con su realidad. En síntesis, nuestra intervención social está anclada, en una determinada dimensión de "lo territorial" (o espacialidad social) y eso, finalmente, es relevante a la hora de poner en marcha procesos de intervención de alto impacto social.

\section{Reflexiones emergentes en torno al debate sobre las palabras y las cosas en Trabajo Social}

Como diría Cortázar (1981), el gran escritor argentino: "Hay palabras que a fuerza de ser repetidas y muchas veces mal empleadas, van perdiendo poco a poco su vitalidad. Y de este modo, en vez de brotar de las bocas o de la escritura como lo que fueron alguna vez, flechas de la comunicación, pájaros del pensamiento y de la sensibilidad, las vemos o las oímos caer como piedras opacas, empezamos a no recibir de lleno su mensaje, o a percibir solamente una faceta de su contenido, a sentirlas como monedas gastadas, a perderlas cada vez más como signos vivos y a servirnos de ellas como pañuelos de bolsillo, como zapatos usados..." (Cortázar, 1981:10). Al parecer, eso ha estado pasando con la palabra intervención/social. Por ello, parece relevante tematizar la intervención social contemporánea y de este modo ir clarificando sus gramáticas, sus actores, sus límites, sus sentidos y fronteras profesionales/disciplinarias. Y en este aspecto re-valoramos lo planteado por Foucault en el siguiente texto: "Puede decirse que es el Nombre el que organiza todo el discurso clásico; hablar o escribir no es decir las cosas o expresarse, no es jugar con el lenguaje, es encaminarse hacia el acto soberano de la denominación, ir, a través del lenguaje, justo hasta el lugar en que las cosas y las palabras se anudan en su esencia común y que permite darles un nombre" (Foucault, 2001:122).

En este contexto, para algunos autores como Moix (2006), la "intervención social" es una expresión inapropiada. Y afirma sin prudencia alguna lo siguiente: "La verdad, en ella no se sabe qué es más desafortunado... si el sustantivo intervención o el adjetivo social (...) Lo de "social" parece obedecer a la necesidad de dejar bien claro, por si alguien lo dudara, que su actividad no se realiza en el mar, o en el aire o en Marte (...) es evidente que no puede actuar más que en el seno de la sociedad en que vive... Lo de intervención social es aún más difícil de justificar, pues en nuestro idioma, esa palabra complica la clara idea de actuar en terreno ajeno, o duplica la clara idea de actuar en terreno ajeno y con fines de regulación o control" (Moix, 2006:276). 
En este contexto analítico, el autor antes reseñado concluye que el Trabajo Social no es la policía, ni el trabajador social un gendarme (aunque siguiendo a Foucault en alguna medida lo es o lo fue). Y como cualquier otro profesional, no interviene, sino actúa.

Frente al planteamiento descrito anteriormente; se hace necesario llamar la atención sobre un aspecto que Moix (2006) ha soslayado en su reflexión, tan radical como ingenua, y que es el hecho de desconocer que en el corazón de la noción de intervención social se halla una determinada relación social. Y que, esta última, por lo menos tiene dos dimensiones (Bajoit, 2009). Por una parte, implica expectativas culturales de los actores y por otra, existen ciertas exigencias o apremios sociales sobre cada uno los implicados en la relación de intervención. En torno a ambas dimensiones se presentan un conjunto de tensiones, cuya resolución no es obvia, automática ni mucho menos mecánica. Y por lo tanto los actores que dan inicio a una relación social de intervención, ya sea por demanda espontánea o por requerimientos institucionales, han debido negociar, tácitamente o explícitamente, unos términos de la interacción, los horizontes de sentido de la misma, las retribuciones esperadas y las contribuciones que cada uno pone en juego en el marco de esta relación social compleja.

En este contexto, toda intervención social es infinitamente más que una simple "actuación profesional", es una praxis social, que articula una acción social y un saber social en una perspectiva transformadora o emancipadora de todas aquellas formas injustas de relación social, de dominación o poder. De lo anterior, emerge un desafío ético y político de contribuir a la constitución de actores sociales capaces de reivindicar sus derechos de ciudadanía. Por otra parte, también surge un desafío técnico y metodológico que implica la necesidad de aportar a esa transformación de nuestras sociedades; específicamente, contribuyendo a la comprensión de ciertos mecanismos de reproducción de la desigualdad social de base en nuestro país.

En conclusión, toda intervención social implica un elemento de poder, entendido como una relación asimétrica entre sujetos concernidos en pos de una acción transformadora. En este sentido, destaca lo señalado por Carballeda (2007), quien precisa la etimología de la palabra intervención como intervenio, alude al acto de "interponerse entre dos cosas". Este significado tendría dos aspectos clave involucrados. Por una parte, es sinónimo de 
mediación, intersección, ayuda o cooperación, Y por otra, nos remite a un acto de intromisión, injerencia, intrusión, coerción o incluso represión. Ambos aspectos de la noción de intervención, son como dos caras de la misma moneda. De allí surge la intervención como un espacio relacional donde se encuentran, artificialmente, dos o más actores sociales que se movilizan hacia determinados horizontes de sentido. De acuerdo a ello, el mismo autor señala que el "reconocer lo artificial de la intervención contribuye a desnaturalizarla y a su vez ayuda a comprenderla como un dispositivo que se entromete en un "espacio relacional problemático" y el cual se articula a partir de una determinada demanda" (Carballeda, 2007:93).

Por último, es preciso reiterar que todo proceso de intervención social involucraen la actualidad un compromiso ético con los actores sociales que están siendo excluidos de los beneficios del desarrollo económico y social en nuestras sociedades latinoamericanas y muy particularmente en el Chile contemporáneo. Por lo tanto, la intervención social no sólo implica la "dramatización" de un rol profesional determinado, ni la puesta en escena de un mero dispositivo técnico de intervención; más bien lo que se activa es una praxis social que está al servicio de generar un tipo de relaciones sociales entre actores/sujetos que favorezcan la construcción de una sociedad justa, solidaria y con mayores niveles de integración social, económica, política y cultural.

\section{Referencias bibliográficas}

Bajoit, G. (1999). La Juventud o el deseo de ser libres. En: Corvalán et al. (Editores). Los jóvenes en Chile y Europa. Educación, trabajo y ciudadanía ( $1^{\text {a }}$ Edición), Santiago: Centro de Investigación y Desarrollo de la Educación.

(2003). Todo cambia. Análisis sociológico del cambio social y cultural en las sociedades contemporáneas ( $1^{\text {a }}$ Ed.), Santiago, Chile: Lom.

(2009). Ser A.S.I de libres en la sociedad contemporánea. Santiago, Chile: S/E.

Carballeda, A. (2007). La intervención en lo social. Exclusión e integración en los nuevos escenarios sociales ( $2^{a}$ ed.). Buenos Aires: Paidós.

Casassus, J. (2003): La escuela y la (des)igualdad ( $1^{\text {a }}$ ed.). Santiago, Chile: Lom. 
CASEN (2006). Encuesta de Caracterización Socioeconómica Nacional. Santiago, Chile: Ministerio de Planificación Nacional.

Castells, M. (2003). La era de la información. Economía, sociedad y cultura. El poder de la identidad. Madrid: Siglo XXI.

CEPAL (2007). Cohesión Social. Inclusión y sentido de pertenencia en América Latina y el Caribe. Santiago, Chile: Comisión Económica para América Latina y el Caribe.

Cortazar, J. (1981). Sobre el cansancio y la enfermedad de las palabras. En: Reunión "Libertad, democracia y derechos humanos", realizada el 26 de marzo de 1981, en Centro Cultural de Villa la Madrid, Argentina.

Foucault, M. (2001). Las palabras y las cosas. Una arqueología de las Ciencias Humanas (10 $0^{\mathrm{a}}$. ed.). México: Siglo XXI.

MIDEPLAN (2007). Serie Análisis de resultados de la Encuesta de Caracterización Nacional [CASEN, 2006]. Distribución del Ingreso e Impacto Redistributivo del Gasto Social, 2006. Santiago, Chile: Ministerio de Planificación.

Moix, M. (2006). La práctica del trabajo social. Madrid: Síntesis.

Molina, W. (2008). El trabajo social chileno frente al desafío de la interculturalidad. Ponencia presentada en el Congreso Ciencias, tecnologías y culturas. Diálogo entre las disciplinas del conocimiento. Mirando al futuro de América Latina y el Caribe. Universidad de Santiago de Chile.

OCDE (2010). Estudio económico de Chile. París: Organization for Economic Cooperation and Development. [En línea|Disponible en http://www.oei.es

OECD/PISA (2006). Evaluación Educativa, resumen ejecutivo. [En línea] Disponible en http://www.oei.es

PNUD (2002). Desarrollo humano en Chile. Nosotros los chilenos: un desafío cultural. Santiago, Chile: CEPAL. 